\title{
Absence of the suprarenal segment of the inferior vena cava with a coexisting absence of the right brachiocephalic vein in a 22-year-old Caucasian male with arterial hypertension
}

\author{
K. Hoffmann ${ }^{1}$, A. Piekarek2 ${ }^{2}$ J. Bieda², K. Karmelita-Katulska², 3, D. Gawrysiak¹, W. Bryl ${ }^{1}$ \\ ${ }^{1}$ Department of Internal Diseases, Metabolic Disorders and Arterial Hypertension, Poznan University of Medical \\ Sciences, Poznan, Poland \\ 2Department of Radiology, Clinical Hospital of Transfiguration, Poznan University of Medical Sciences, Poznan, Poland \\ ${ }^{3}$ Department of Neuroradiology, Poznan University of Medical Sciences, Poznan, Poland
}

[Received 11 February 2014; Accepted 11 July 2014]

Congenital anomalies of the inferior vena cava (IVC) are rarely observed malformations of the venous system, occurring in $0.3 \%$ of otherwise healthy individuals, and in $0.6 \%$ to $2 \%$ of patients with coexisting cardiovascular defects. They are usually asymptomatic and recognised incidentally during imaging, operations or dissection studies. In this paper we report an extremely rare case of a 22-year-old Caucasian male, admitted for the purpose of excluding secondary causes of hypertension. During imaging of the abdomen and the thorax we found a complete lack of the suprarenal segment of the IVC, with a coexisting absence of the right brachiocephalic vein. We discuss the problem of congenital defects of the IVC and we review the relevant literature. (Folia Morphol 2015; 74, 1: 122-126)

Key words: congenital anomaly, inferior vena cava, systemic veins

\section{INTRODUCTION}

Anomalies of the inferior vena cava (IVC) are very rare malformation of the venous system; therefore data about the clinical presentation of patients are scarce. It is estimated that they occur in $0.3 \%$ of otherwise healthy individuals, and in $0.6 \%$ to $2 \%$ of patients with coexisting various cardiovascular abnormalities, although these figures likely represent underestimates [15]. Congenital anomaly of the IVC may be a stand-alone defect or it may coexist with disturbances in the development of other vessels and organs, especially the heart and the spleen $[7,10,17]$. Generally, the majority of the anatomical anomalies of the IVC are usually recognised incidentally during imaging, operations or dissection studies. Except for the interruption of the IVC, which is recognised more often in females, they are observed mostly in males $(62 \%)$ and its prevalence is similar in Asiatic, European and other Western countries, with the exception of aplasia/agenesis of the IVC, which is relatively less prevalent in Asia [8]. More prevalent anatomical variants within the spectrum of the IVC malformations are: the duplicate IVC $(0.2-3 \%)$, transposition (left-sided IVC $0.2-0.5 \%$ ), the circumaortic left renal vein $(1.5-8.7 \%)$ and the retro-aortic renal vein $(2.1 \%)$ [8]. Rare variations of the IVC include the absence of a part of the IVC, azygos and hemiazygos continuation of a double IVC, hypoplasia, or aplasia/agenesis, or interruption of the IVC, double superior vena cava (SVC) and double IVC [16].

In this study we report a case of a young Caucasian male with arterial hypertension (HT), in whom

Address for correspondence: K. Hoffmann, MD, PhD, Chair and Department of Internal Diseases, Metabolic Disorders and Arterial Hypertension, Poznan University of Medical Sciences, ul. Szamarzewskiego 84, 60-569 Poznań, Poland, tel: +48 618549377 , fax: +48 61 8478529 , e-mail: karhof@tlen.pl 
asymptomatic abnormalities of the venous system were found, both in the thorax and in the abdomen.

\section{CASE REPORT}

A 22-year-old male patient was admitted to the Department of Arterial Hypertension and Metabolic Disorders for the purpose of excluding secondary causes of HT. He had a history of HT, diagnosed a few months before hospitalisation and treated with a small dose of beta-blocker (bisoprolol $1.25 \mathrm{mg}$ once a day), and his family history was remarkable for essential HT and premature coronary heart diseases. On the physical examination his blood pressure was $120 / 80 \mathrm{~mm} \mathrm{Hg}$, pulse rate was $95 \mathrm{bpm}$, and respiratory rate was 14 breathes per minute. His electrocardiogram was normal sinus rhythm. Laboratory study results were essentially normal except for a mildly elevated serum alanine aminotransferase level (57 IU/L; normal level below $45 \mathrm{IU} / \mathrm{L}$ ), asparagine aminotransferase level (42 IU/L; normal level below $35 \mathrm{IU} / \mathrm{L}$ ) and microalbuminuria $(125.6 \mathrm{mg} / 24 \mathrm{~h}$; the range of microalbuminuria is $30-300 \mathrm{mg} / 24 \mathrm{~h}$ ). We also estimated plasma renin activity, serum aldosterone concentration, metoxycatecholamines in two 24-h urine samples - the results were negative. Ambulatory blood pressure monitoring showed mean circadian blood pressure $136 / 82 \mathrm{~mm} \mathrm{Hg}$ and dipping status of the patient. Transthoracic echocardiography revealed normal size and good systolic function of the left ventricle, no pathological valvular flows and no other abnormalities. Ultrasound imaging of the abdomen showed normal kidneys and suprarenal glands. Finally, on the base of performed diagnostics, we excluded secondary causes of HT.

However, during routine ultrasonography of the abdominal cavity with the option of Doppler, we found a complete lack of the suprarenal segment of the IVC, with coexisting widening of the portal vein $(1.2 \mathrm{~cm})$ and splenomegaly $(16 \mathrm{~cm})$. Two hepatic veins were visualised, but it was not clear if they went directly to the right atrium or if there was a short intrahepatic part of the IVC. Ultrasound imaging of the portal vein drainage was difficult, the hilar part of the portal vein was widened $-1.2 \mathrm{~cm}$, but there were no signs of thrombosis. As the next step, computed tomography angiography (CTA) was performed, allowing visualising the agenesis of the suprarenal segment of the IVC (Fig. 1). There were no abnormalities in the number and course of hepatic veins. There were 3 of them and they all went to the short intrahepatic part of the IVC and then to the right

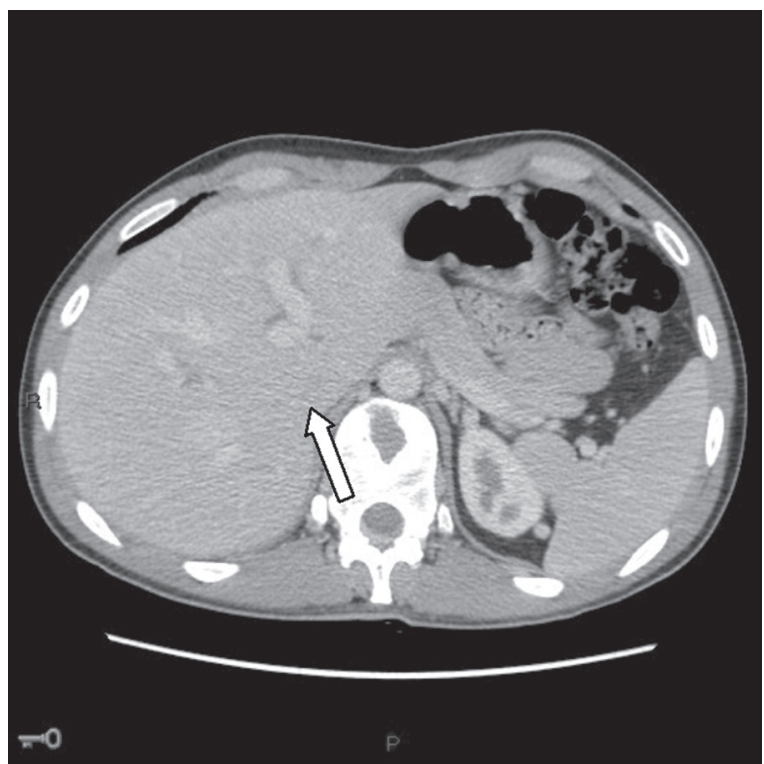

Figure 1. Absence of the suprarenal portion of the interior vena cava (computed tomography) — section at the portal level of the liver.

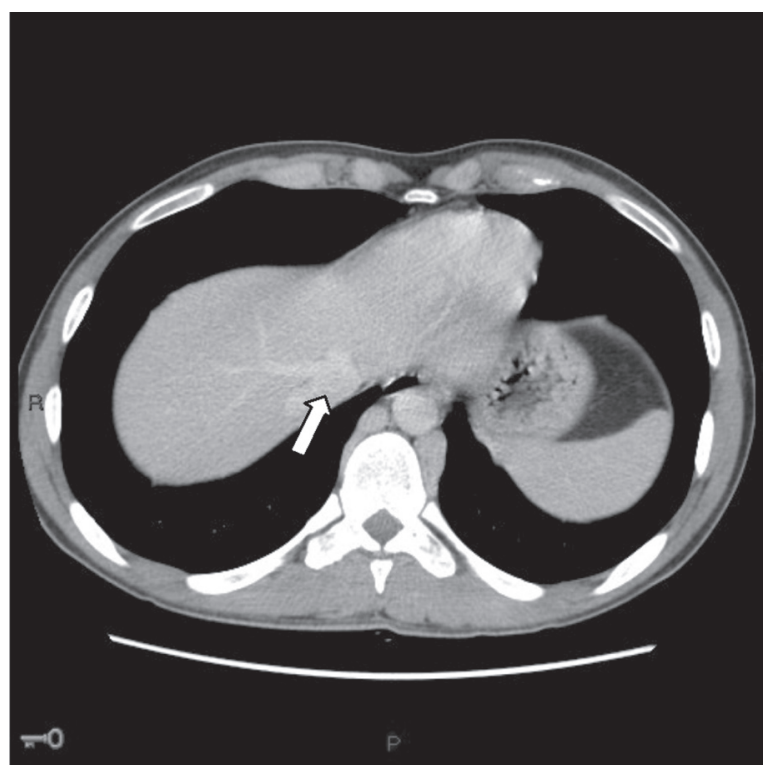

Figure 2. Well-visualised segment of the inferior vena cava before draining into the right atrium (computed tomography).

atrium (Fig. 2) CTA also revealed no abnormalities in portal venous system. Splenomegaly was present. In CTA it was able to see a well-developed collateral circulation, formed by the renal veins, the lumbar veins, the anterior vertebral venous plexuses and the azygos veins (Figs. 3, 4). No other vessel malformations and organ anomalies of the abdomen and the pelvis were confirmed. The presence of renal and infrarenal part of the IVC was shown on Figure 3. 


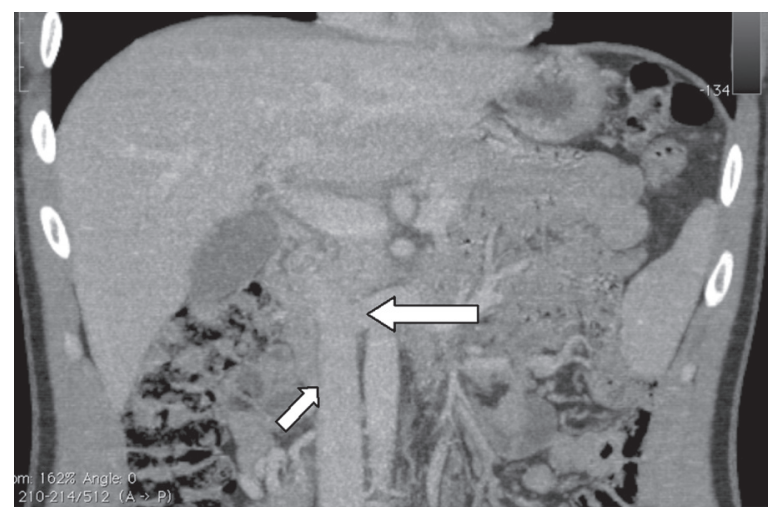

Figure 3. The renal (the big arrow) and infrarenal portion (the small arrow) of the inferior vena cava (computed tomography).

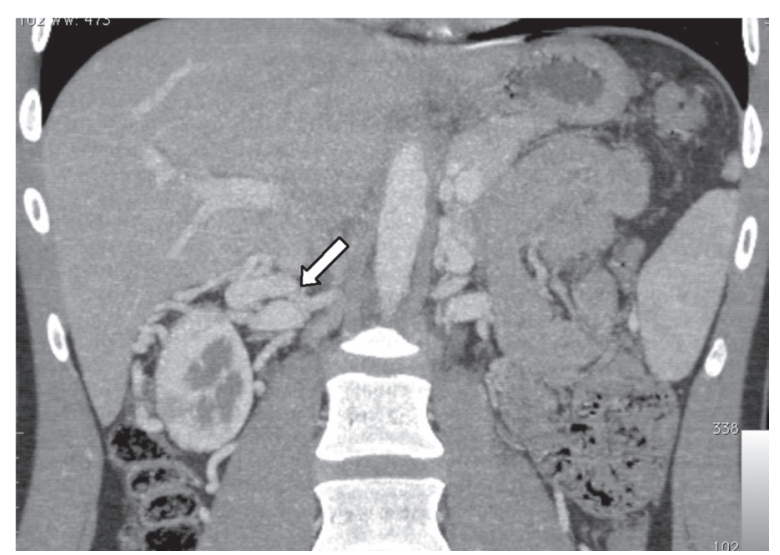

Figure 4. Collaterals from the right renal vein (computed tomography).

In order to complete the diagnostics, we performed magnetic resonance imaging (MRI) angiography of the thorax, which revealed the presence of widened azygos vein as the consequence of collateral circulation (Fig. 5). MRI also showed coexisting agenesis of the right brachiocephalic vein and ipsilateral internal jugular vein (Fig. 6). In the superior aperture of the thorax there was a vast vascular network which drained into the right subclavian vein, and then via the perivertebral vessels to the left subclavian vein. We confirmed that on the right side the subclavian vein drained directly to the left subclavian vein. MRI of the thorax revealed no anatomical abnormalities in SVC, venous drainage on the left side and arterial system.

Since there was no need for surgical intervention, the patient was discharged in a very good condition. During 1 year follow-up the patient was leading normal life and no significant health problems were noted.

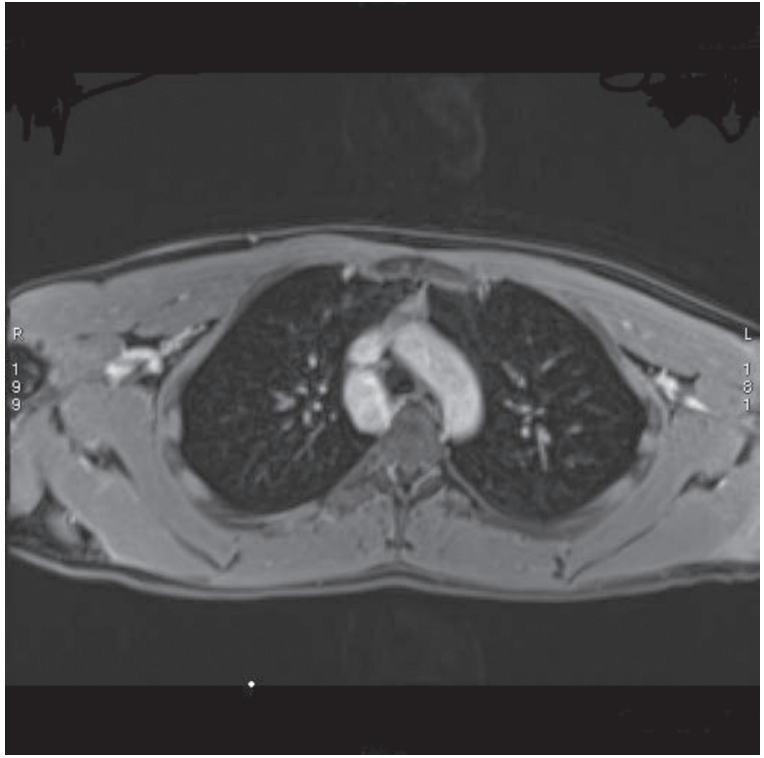

Figure 5. Wide azygos vein (magnetic resonance imaging).

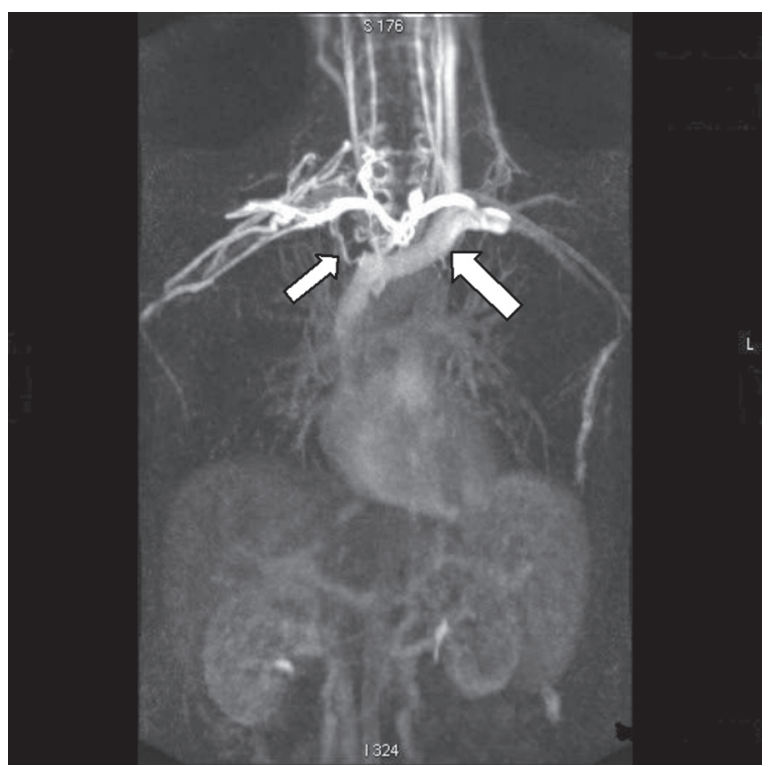

Figure 6. Absence of the right brachiocephalic vein (the small arrow), existing left brachiocephalic vein (the big arrow). The early phase (magnetic resonance imaging).

\section{DISCUSSION}

Embrylogically, there are 4 segments of normal IVC: hepatic, suprarenal, renal and infrarenal. The development of these segments, occurring in the period between the $4^{\text {th }}$ and $8^{\text {th }}$ gestational week, is a very complicated process, which could be disturbed by various factors and finally result in various anomalies of the IVC. The hepatic segment of the IVC is formed 
by the vitelline vein. Primarily, there are 3 pairs of embryonic veins that play a crucial role in the developmental process of the vena cava system: posterior cardinal, which appear first and regress, subcardinal and supracardinal. At embryologic period this bilateral cardinal venous system provides the main venous drainage of the foetus and establishes the connection between systemic and pulmonary veins. Subsequently, vena cava system generally is formed as the anastomoses between cardinal veins developed and pulmonary veins are linked to left atrium. Cardinal veins give rise to vena cava respectively: the right anterior vein forms the SVC, whereas 2 segments of the IVC develops from the right supracardinal vein (the infrarenal segment of IVC) and from the right subcardinal vein (the suprarenal segment of IVC). The renal part of the IVC is derived from the right suprasubcardinal and post-subcardinal anastomoses. In the abdominal region, the post-cardinal veins are progressively replaced by the supracardinal and subcardinal veins, whereas in the pelvis they persist as the common iliac veins. In the thorax, the azygos and hemiazygos veins develops from the supracardinal veins $[2,16]$. The right brachiocephalic vein, which was not visualised in this patient, normally is formed from the connection of 2 vessels: the internal jugular vein and the subclavian vein. The aberrant development of the IVC may lead to an increased incidence of intrahepatic and extrahepatic shunts between systemic and portal veins, which should be surgically corrected $[5,6]$.

The diagnosis of agenesis of the IVC is quite challenging. In general, it is mostly incidentally diagnosed during surgeries, or through imaging of the area, usually with $\mathrm{CT}$ scanning or MRI done for other reasons [1, 2]. If a venous collateral circulation is efficient enough, this anomaly is usually asymptomatic [11, 13]. In the literature some clinical manifestations of the disease have been described, such as isolated iliac vein thrombosis in small children and young subjects, varicose veins of the internal organs and intravertebral venous plexus, pathological mediastinal or abdominal masses, cyanosis of the abdomen and lower extremities $[5,7,10,17]$.

It is interesting to note that the presence of the IVC anomalies seems to be a predisposing factor for thromboembolic diseases and pulmonary embolism. In the study conducted by Ruggeri et al. [14] it was estimated that $5 \%$ of young patients with idiopathic deep vein thrombosis had a congenital malformation of the IVC. Moreover, it was suggested that there could be a link between congenital anomalies of the IVC and thrombophilia disorders. Actually, the screening for thrombophilia in subjects with the IVC malformations is still incomplete, resulting in inability to assess the true prevalence of thrombophilia in congenital anomalies of the IVC $[3,9,13,14]$.

It must be emphasised that in patients with any abnormalities of the IVC, concomitant anatomical abnormalities should be considered. What is more, it is suggested to examine first-degree relatives as they are at risk of congenital anomalies of the vascular system [12]. In the presented case, family history was remarkable for essential HT and premature coronary heart diseases, but not for any vascular abnormalities. In addition, no defects of the heart were found in the subject; however some types of the IVC malformations were described in patients with congenital cardiac anomalies [1]. We also did not detect any serious renal anomalies, except for a cyst $(17 \mathrm{~mm})$ in the right kidney, whereas Gayer et al. [4] reported right renal aplasia or hypoplasia in a small series of patients with IVC anomalies.

\section{CONCLUSIONS}

In closing, it is worth paying attention to 3 main conclusions. Firstly, although anomalies of the IVC are extremely rare, they should be taken into account during retroperitoneal surgeries in order to avoid significant complications, especially venous haemorrhage caused by mistaken injury of the IVC. A precise knowledge of anatomical variation of the IVC is crucial during such operations as ureteral surgery, sympathectomy, radical nephrectomy and organ transplantation. Secondly, the clinical importance of these anomalies lies also in the potential for misdiagnosis during imaging. Thirdly, they are of great importance in the diagnosis and the treatment of venous thromboembolic disease and pulmonary embolism. Clinicians should pay attention to those patients with thromboembolic diseases in whom they want to place a filter in the vena cava. In case of unrecognised anomaly of the IVC, e.g. double IVC or segmental absence of IVC, there is a great risk of recurrence of embolisms [8] or severe complications during the procedure. It should be underlined that all the congenital malformations of the IVC may cause recurrent deep vein thrombosis, especially in young patients. 


\section{REFERENCES}

1. Abdullah A, Williamson K, Lewis T, Elsamaloty H (2011) Variant central intrahepatic course of interior vena cava: volume-rendering and maximum intensity projection $\mathrm{CT}$ findings. Br J Radiol, 84: e135-e137.

2. Chuang VP, Mena CE, Hoskins PA (1974) Congenital anomalies of the inferior vena cava. Review of embryogenesis and presentation of a simplified classification. Br J Radiol, 47: 206-213.

3. Gayer G, Luboshitz J, Hertz M, Zissin R, Thaler M, Lubetsky A, Bass A, Korat A, Apter S (2003) Congenital anomalies of the interior vena cava revealed on $\mathrm{CT}$ in patients with deep vein thrombosis. Am J Roentgenol, 180: 729-732.

4. Gayer G, Zissin R, Strauss S, Hertz M (2003) IVC anomalies and right renal aplasia detected on CT: a possible link? Abdominal Imaging, 28: 395-399.

5. Guérin F, Blanc Th, Gauthier F, Franchi Abella S, Branchereau S (2012) Congenital portosystemic vascular malformations. Seminars in Pediatric Surgery, 21: 233-244.

6. Guo FF, Shao ZQ, Li HL, Wang GJ, Zhu WB (2011) Successful use of the dilated right spermatic cord vein for renal transplantation in a patient with congenital hypoplasia of the interior vena cava. Transplantation Proceeding,43: 4002-4004.

7. Joskin J, Bleus N, Couvreur T, Tselikas L, Milicevic M (2012) Duplicate inferior vena cava continuing as a hemiazygos continuation draining into the right atrium via persistent left superior vena cava. JBR-BTR, 95: 174.

8. Kapetanakis S, Papadopoulos C, Galani P, Dimitrakopoulou G, Fiska A (2010) Anomalies of the interior vena cava: a report of two cases and a short review of the literature. Folia Morphol, 69: 123-127.
9. Kouroukis Ch, Leclerc JR (1996) Pulmonary embolism with duplicated vena cava. Chest, 109: 1111-1113.

10. Mutlu GY, Ramot Y, Babaoglu K, Altun G, Zlotogorski A, Molho-Pessach V (2013) Agenesis of the inferior vena cava in $\mathrm{H}$ syndrome due to a novel SLC29A3 mutation. Pediatric Dermatol, 30: e70-e73.

11. Nseir W, Mahamid M, Abu-Rahmed Z, Markel A (2011) Recurrent deep venous thrombosis in a patient with agenesis of inferior vena cava. Int J Gen Med, 4: 457-459.

12. Obernosterer A, Aschauer M, Mitterhammer H, Lipp RW (2004) Congenital familial vascular anomalies: a study of patients with an anomalous inferior vena cava, and of their first degree relatives. Angiology, 55: 73-77.

13. Obernosterer A, Aschauer M, Schnedl W, Lipp RW (2002) Anomalies of the interior vena cava in patients with iliac venous thrombosis. Ann Intern Med, 136: 37-41.

14. Ruggeri M, Tosetto A, Castaman G, Rodeghiero F (2001) Congenital absence of the inferior vena cava: a rare risk factor for idiopathic deep-vein thrombosis. Lancet, 357: 441.

15. Timmers GJ, Falke TH, Rauwerda JA, Huijgens PC (1999) Deep vein thrombosis as a presenting symptom of congenital interruption of the inferior vena cava. Int J Clin Pract, 53: 75-76.

16. Tore HG, Tatar I, Celik HH, Oto A, Aldur MM, Denck CC (2005) Two cases of interior vena cava duplication with their $\mathrm{CT}$ findings and a review of literature. Folia Morphol, 64: 55-58.

17. Yugueros $X$, Alvarez B, Fernandez E, Boque $M$, Matas $M$ (2013) Compressive symptoms due to thrombosed or hypertrophic collateral circulation in infrarenal inferior vena cava agenesis. Ann Vasc Surg, 27: 238.e9-238.e13. 